\title{
Analysis of Pollution and Blockage of Titanium Rod Sintered Microporous Filter Element
}

\author{
Ronglei Xiong ${ }^{*}$, Peng Liu ${ }^{1}$, Yaoyuan Zhang², Yuan Nan², Jinding Chen ${ }^{2}$, Xiaojuan Chen ${ }^{2}$, \\ Shuangzheng $\mathrm{Ma}^{2}$, Guanqiao Lin², Qun Zhang² \\ ${ }^{1}$ Zhanjiang Branch of CNOOC (China) Co., Ltd., Zhanjiang, China \\ ${ }^{2}$ Engineering Technology Branch of CNOOC Energy Development Co., Ltd., Zhanjiang, China \\ Email: *1057155090@qq.com
}

How to cite this paper: Xiong, R.L., Liu, P., Zhang, Y.Y., Nan, Y., Chen, J.D., Chen, X.J., Ma, S.Z., Lin, G.Q. and Zhang, Q. (2021) Analysis of Pollution and Blockage of Titanium Rod Sintered Microporous Filter Element. Open Journal of Applied Sciences, 11, 614-622.

https://doi.org/10.4236/ojapps.2021.115044

Received: April 28, 2021

Accepted: May 25, 2021

Published: May 28, 2021

Copyright $\odot 2021$ by author(s) and Scientific Research Publishing Inc. This work is licensed under the Creative Commons Attribution International License (CC BY 4.0).

http://creativecommons.org/licenses/by/4.0/

\begin{abstract}
Microfiltration is widely used in fine filtration operations, with dead-end filtration and cross-flow filtration. The microfiltration filter element or the microfiltration membrane is easy to be polluted by impurities in the water and causes fouling, resulting in flux attenuation. The flux can be expressed by Darcy's law and attenuation model. In this paper, two industrial titanium rod sintered filter elements $(\varnothing 60 \times 960 \mathrm{~mm}$ and $\varnothing 60 \times 960 \mathrm{~mm})$ of different specifications are selected, and tap water (1.0 NTU) is used for constant pressure dead-end filtration. The amount tends to be the same, about $0.435 \mathrm{~m}^{3} \cdot \mathrm{m}^{-2} \cdot \mathrm{h}^{-1}$, which has nothing to do with the filtration accuracy of the filter element but only depends on the characteristics of the filter cake and the filter membrane. Through the analysis of the two models, it is found that the two filtration flux models are not universal and difficult to be applied in engineering.
\end{abstract}

\section{Keywords}

Microfiltration, Titanium Rod Sintered Filter Element, Membrane Pollution, Cleaning

\section{Introduction}

Microporous filtration uses the pore size of the microporous membrane to trap particles, bacteria, and suspended substances that are larger than the pore size of the microporous membrane in the filtrate under the pressure difference, so as to achieve the purpose of removing the particulates and clear solution in the filtrate. Generally, the pore size of the microporous membrane used in the micro-

${ }^{\star}$ First author. 
filtration process is in the range of $0.05-10 \mu \mathrm{m}$ [1]. Microporous filtration is basically a solid-liquid separation operation, without considering the influence of concentration polarization and solution osmotic pressure. Microfiltration operation the pressure difference is about $0.01-0.2 \mathrm{MPa}[2]$, and the permeation flux of the membrane is much greater than that of ultrafiltration, nanofiltration, and reverse osmosis. Ultrafiltration, nanofiltration, and reverse osmosis are mainly used for homogeneous separation operations to remove solutes from the solution.

\section{Microfiltration Operation Mode and Theoretical Basis}

\subsection{Microfiltration Operation Mode}

The microfiltration process has two operation modes: terminal microfiltration and cross-flow microfiltration, as shown in Figure 1. In the terminal microfiltration operation, the trapped particles continue to form a filter cake on the membrane surface, and continue to thicken with the filtration time, so that the resistance of the microfiltration continues to increase. If the pressure drop is kept constant, the membrane flux will be caused. Continue to decline, if the membrane flux is kept constant, the microfiltration thrust needs to be continuously increased. Therefore, the terminal microfiltration is usually intermittent, and the filter cake or the filter membrane must be periodically removed during the process.

Cross-flow microfiltration is based on the sieving mechanism of membrane surface filtration. The operation mode is to use a pump to send the filtrate into a pipe or thin-layer flow channel with a porous membrane wall. The filtrate flows along the tangential direction of the membrane surface. Under the driving force, permeate is cross-flowed through the membrane. As shown in Figure (b), convective mass transfer brings particles to the membrane surface and deposits to form a thin filter cake. Unlike terminal microfiltration, the filter cake layer in the cross-flow microfiltration process does not thicken indefinitely. On the contrary, the shear force generated by the tangential flow of the material liquid on the membrane surface can wash away some particles deposited on the membrane surface, so the thickness of the filter cake layer accumulated on the membrane surface is relatively thin. Since the cross-flow operation can effectively control the concentration polarization and the thickening of the filter cake, it can maintain a relatively high flux for a long period of time. Once the thickness of the filter cake layer is stable, the flux also reaches a steady state or pseudo steady state [3].

\subsection{Mathematical Model of Filter Element Filtration}

There are many microporous filtration models, of which two typical mathematical models have been widely recognized by scholars, namely the filter cake filter flux model and the microporous filter flux attenuation model. The core expressions are shown in Table 1. 
Table 1. Two mathematical models of microfiltration operation.

Conventional filter cake filtration flux model

Filter flux: $J=\frac{1}{A} \times \frac{\mathrm{d} V}{\mathrm{~d} t}=\frac{\Delta p}{\mu\left(R_{\mathrm{m}}+R_{\mathrm{c}}\right)}$

Membrane resistance: $R_{\mathrm{m}}=\frac{k_{\mathrm{m}}\left(1-\varepsilon_{\mathrm{m}}\right)^{2} S_{\mathrm{m}} l_{\mathrm{m}}}{\varepsilon_{\mathrm{m}}^{3}}$

Cake resistance: $R_{\mathrm{c}}=\frac{k_{\mathrm{c}}\left(1-\varepsilon_{\mathrm{c}}\right)^{2} S_{\mathrm{c}} l_{\mathrm{c}}}{\varepsilon_{\mathrm{c}}^{3}}$

$J$-Filtration flux (also called membrane filtration rate), $\mathrm{m}^{3} \cdot \mathrm{m}^{-2} \cdot \mathrm{h}^{-1}$;

$A$-Filter area, $\mathrm{m}^{2}$;

$V$-Permeate filtrate volume, $\mathrm{m}^{3}$;

t-Filter time, h;

$\Delta p$-The pressure difference applied to the membrane and the filter cake, $\mathrm{kPa}$;

$\mu$-Viscosity of the suspension fluid, Pa.s;

$R_{\mathrm{m}}, R_{\mathrm{c}}$-Respectively the resistance of the membrane layer and the filter cake layer, $N$;

$k_{\mathrm{m}}, k_{\mathrm{c}}$-Respectively filter membrane constant $\left(k_{\mathrm{m}}=2\right)$ and filter cake constant $\left(k_{c}=5\right)$;

$\varepsilon_{\mathrm{m}}, \varepsilon_{\mathrm{c}}$-Respectively, membrane porosity and filter cake porosity, dimensionless,

$S_{\mathrm{m}}, S_{\mathrm{c}}$-Respectively, the specific surface area of the filter membrane and the specific surface area of the filter cake, $\mathrm{m}^{3} \cdot \mathrm{m}^{-2}$;

$I_{\mathrm{m}}, I_{\mathrm{c}}$-Respectively the thickness of the filter membrane and the thickness of the filter cake, $\mathrm{m}$.
Microporous filtration flux attenuation model

(1) Filter flux: $J=\frac{1}{A} \times \frac{\mathrm{d} V}{\mathrm{~d} t}=\frac{\beta}{(\alpha t+\beta)^{2}} \times \frac{1}{A}$

(2) Model parameters: $\alpha=\frac{4}{\pi h_{\mathrm{m}} n_{\mathrm{p}} d_{\mathrm{p}}^{2}} \times \frac{N}{A \rho_{\mathrm{s}}(1-\varepsilon)}$

(3) Model parameters: $\beta=\frac{h_{\mathrm{m}}}{N} \times \frac{128 \mu}{\pi d_{\mathrm{p}}^{2} A \times \Delta p_{\mathrm{TMP}}}$

$J$-Filtration flux (also called membrane filtration rate), $\mathrm{m}^{3} \cdot \mathrm{m}^{-2} \cdot \mathrm{h}^{-1}$;

$A$-Filter area, $\mathrm{m}^{2}$;

$V$-Permeate filtrate volume, $\mathrm{m}^{3}$;

$t$-Filter time, h;

$\alpha, \beta$-Model parameters;

$\mu$-Viscosity of the suspension fluid, Pa.s;

$h_{\mathrm{m}}$-Circulation depth, m;

$n_{\mathrm{p}}$-Number of membrane holes;

$d_{\mathrm{p}}$-Porous outer diameter before filtration, $\mathrm{m}$;

$\mathrm{N}$-Concentration of suspended solids entering the porous, $\mathrm{kg} \cdot \mathrm{m}^{-3}$;

$\mathcal{E}$-The porosity of the pore surface layer structure is dimensionless,

$\Delta p_{\text {TMP }}$ - The pressure difference applied to the membrane and the filter cake, $\mathrm{kPa}$;

$\rho_{\mathrm{s}}$-Solid particle density, $\mathrm{kg} \cdot \mathrm{m}^{-3}$.

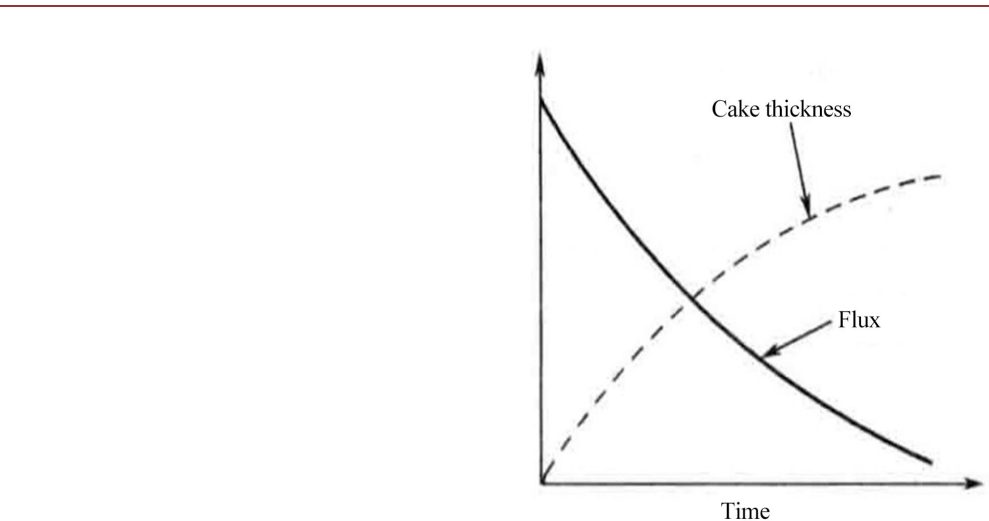

(a)

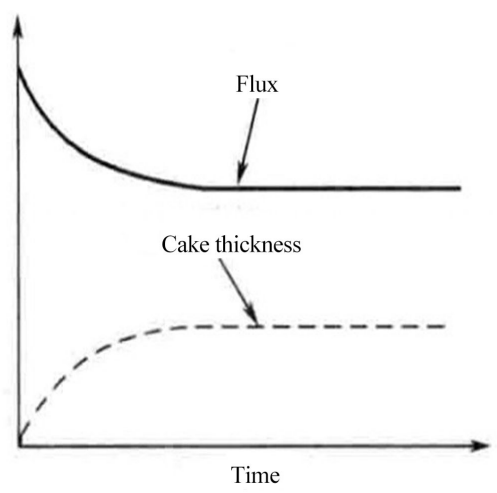

(b)

Figure 1. Two filtration operation modes of microfiltration. (a) Terminal filtering (deadend filtering); (b) Cross-flow filtration (dynamic filtration).

\section{Test Experiment of Filtration Flux of Titanium Rod Sintered Filter Element}

\subsection{Filtration Flux Test Device}

The filtration flux test device is shown in Figure 2. The device can realize two test modes of constant pressure and constant flow to investigate the influence of different pressures and different flows on the pollution rate of the filter element. 


\subsection{The Test Results of the Final Filtration of Different Specifications of the Great Filter Element}

The titanium rod filter element is provided by Shijiazhuang Gold Titanium filter element, and its specifications are $\varnothing 60 \times 960 \mathrm{~mm}$ and $\varnothing 60 \times 960 \mathrm{~mm}$, and the filtration area of a single rod is $0.181 \mathrm{~m}^{2}$. When the flow rate is greater than 0.2 $\mathrm{m}^{3} / \mathrm{h}$, use an electromagnetic flowmeter to read, and when the flow rate is less than $0.2 \mathrm{~m}^{3} / \mathrm{h}$, use an electronic scale and a stopwatch to measure. The turbidity of tap water is $1.0 \mathrm{NTU}$, and the turbidity is $0 \mathrm{NTU}$ after filtering by the filter element. The flux attenuation trend is as follows shown in Figure 3.

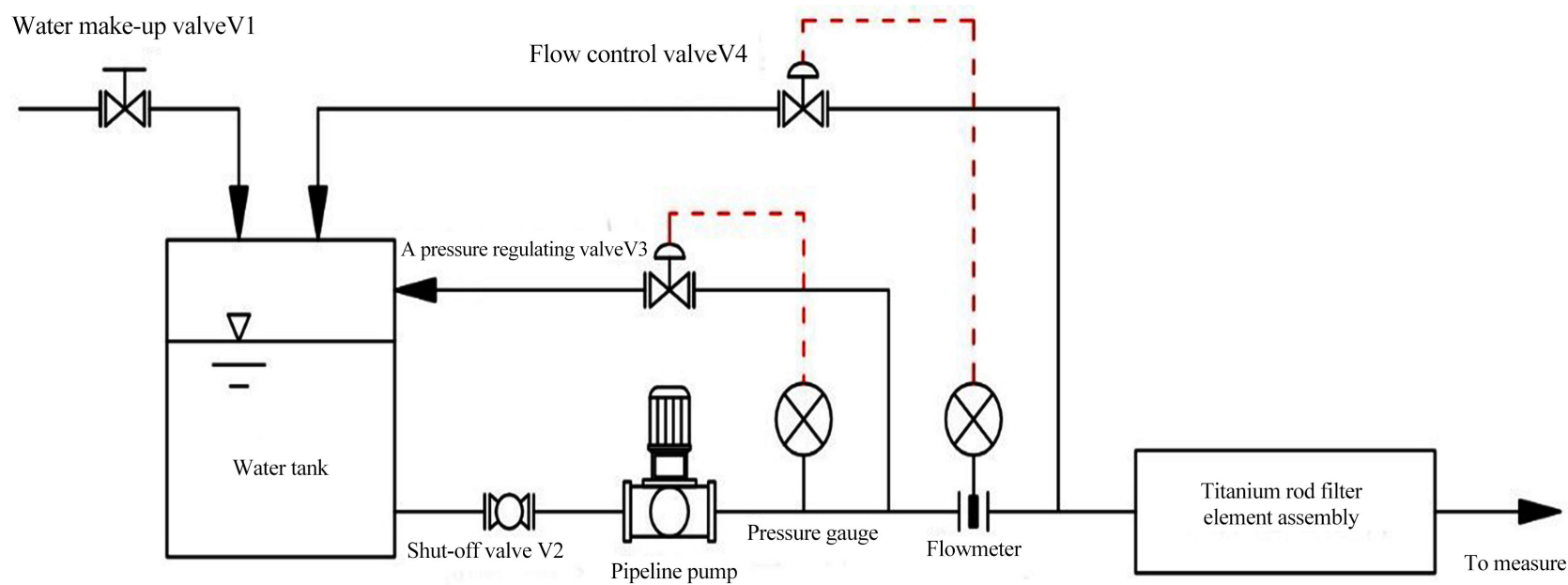

Figure 2. Flow of filter element flux test device.

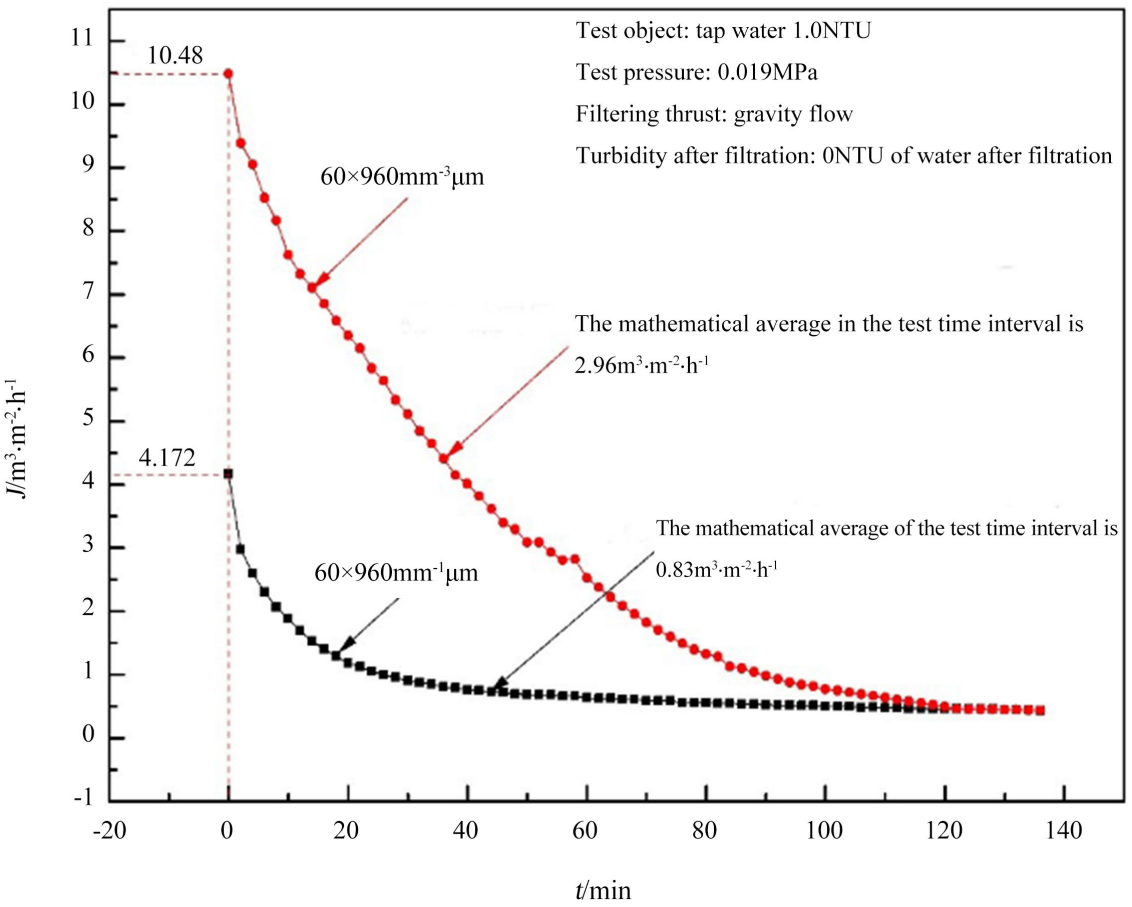

Figure 3. $\varnothing 60 \times 960 \mathrm{~mm}$ and $\varnothing 60 \times 960 \mathrm{~mm}$ titanium rod filter element constant pressure terminal filtration flux attenuation trend. 
The mathematical average of the flux in the test interval is obtained by the Origin scatter integral, namely:

$$
\bar{J}=\frac{1}{\Delta t} \int_{t_{1}}^{t_{2}} J \mathrm{~d} t
$$

Know from Figure 3:

1) As the filtration progresses, the filtration resistance continues to increase, the filter element continues to be contaminated, and the flux continues to decrease. The flux decreases rapidly at the beginning of the filtration, and gradually slows down after the filter cake is formed;

2) Large pores have a large flux, the filtration resistance increases faster, the fouling speed is fast, and the flux decays fast. The small pores have a small flux, the filtration resistance increases relatively slowly, and the fouling speed also slower;

3) Whether it is a $1 \mu \mathrm{m}$ or $3 \mu \mathrm{m}$ filter element, the flux basically tends to be the same after $2 \mathrm{~h}$. Therefore, it can be inferred that the ability to intercept suspended solids after the curve enters the flat section is basically independent of the micropore diameter, and the backwash cycle should not exceed $2 \mathrm{~h}$.

\section{Analysis of Constant Pressure Dead End Filtration Model}

\subsection{Filter Cake Filter Flux Model}

In this article, tap water is filtered with micropores. Tap water contains both organic matter and minerals, as well as microorganisms and other impurities. During filtration, a gel layer is formed near the surface of the membrane. This gel particle has a certain degree of elasticity. The particles deform greatly and enter the microporous pore channel under pressure, which not only thickens the filter cake layer on the surface of the filter membrane, but also passes through the adsorption bridging and salting out in the gap after entering the pore channel of the filter membrane, which affects the filtration The factors are complicated. Both the filter membrane resistance $R_{m}$ and the filter cake resistance $R_{c}$ are no longer constants, and it is difficult to use a reliable formula for effective expression. Therefore, the filter cake filtration flux model is rarely used in engineering design.

\subsection{Establishment of Flux Decay Model Parameters}

The pre-expression of the flux decay model is:

$$
\frac{t}{V}=\alpha t+\beta
$$

Rearrange and differentiate the formula (7) to obtain the formula (4) in Table 1. Now the test data in Figure 3 is sorted according to the formula (7) and then fitted to the result shown in Figure 4.

Obviously, the relationship between $t / V \sim t$ is not a straight line, that is, $\alpha$ and $\beta$ are not constants as stated in the model, but a variable that changes with the 


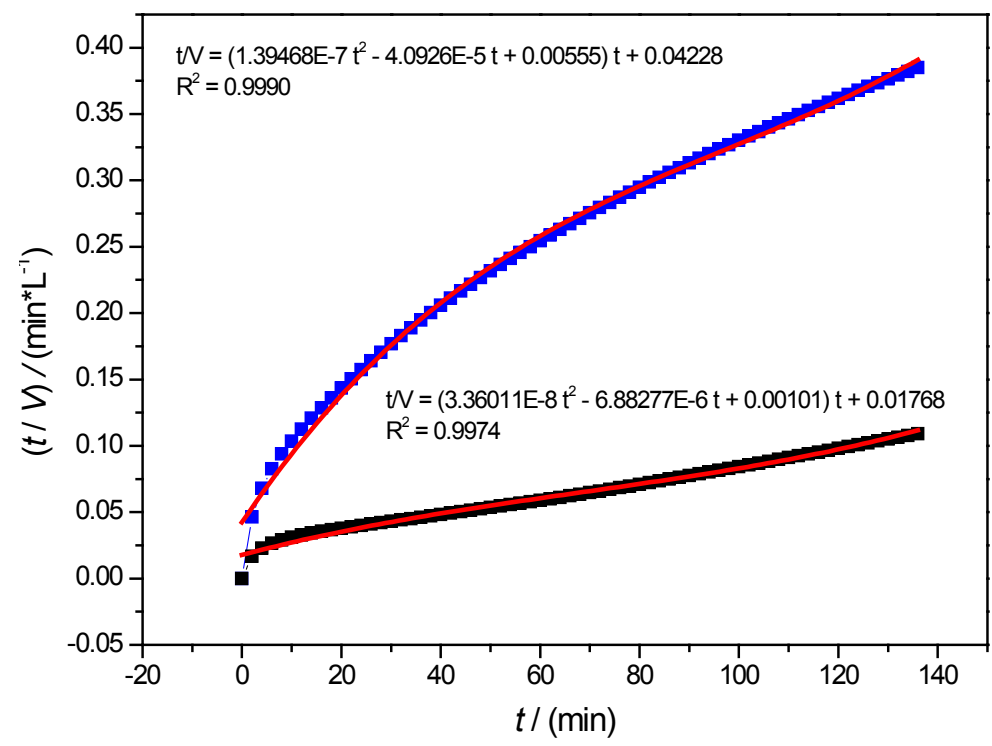

Figure 4. Fitting results of the test data of two specifications of filter elements according to the attenuation model.

filtering time. Not only that, the pore size of the filter element is different, and the shape of the attenuation curve is also different, so the model does not have universality and versatility.

$$
\begin{gathered}
\alpha_{1}=1.39468 \times 10^{-7} t^{2}-4.0926 \times 10^{-5} t+0.00555, \beta_{1}=0.04228, R^{2}=0.9990 \\
(\varnothing 60 \times 960 \mathrm{~mm}) \\
\alpha_{2}=3.36011 \times 10^{-8} t^{2}-6.88277 \times 10^{-6} t+0.00101, \beta_{2}=0.01768, R^{2}=0.9974 \\
(\varnothing 60 \times 960 \mathrm{~mm})
\end{gathered}
$$

Substituting the obtained model parameters into Equation (4):

$$
\begin{aligned}
& J_{1}=\frac{0.04228}{\left[\left(1.39468 \times 10^{-7} t^{2}-4.0926 \times 10^{-5} t+0.00555\right) t+0.04228\right]^{2}} \times \frac{1}{0.181} \\
& J_{2}=\frac{(\varnothing 60 \times 960 \mathrm{~mm})}{\left[\left(3.36011 \times 10^{-8} t^{2}-6.88277 \times 10^{-6} t+0.00101\right) t+0.01768\right]^{2}} \times \frac{1}{0.181} \\
& (\varnothing 60 \times 960 \mathrm{~mm})
\end{aligned}
$$

Two kinds of filter elements need two different model parameters to express. Obviously, this has lost the meaning of the model, so the method of experimental determination is still used in engineering.

\section{Unblocking of Titanium Rod Sintered Filter Element}

Titanium rod sintered filter element is made of industrial pure titanium powder (purity $>99.7 \%$ ) as the main raw material, sintered at high temperature, and the thickness of the microporous film is generally $90-170 \mu \mathrm{m}$. Its uniform structure, high porosity, low filtration resistance, good permeability, high filtration precision (up to $0.1 \mu \mathrm{m}$ ), acid and alkali corrosion resistance, high temperature 
resistance, etc., can be widely used in the separation and purification of solid-liquid and solid-gas. It is especially suitable for pre-filtration of ultrafiltration, nanofiltration and reverse osmosis.

\subsection{Pollution of Titanium Rod Filter Element}

Membrane fouling is usually caused by the formation of an adhesion layer on the membrane surface and the blockage of the membrane channels [4] [5] [6]. When the solute is a water-soluble macromolecule, because its diffusion coefficient is very small, the diffusion flux from the membrane surface to the main body of the liquid is also very small, so the solute concentration on the membrane surface is significantly increased to form an immobile gel layer. The adhesion layer on the surface of the membrane may also be an adsorption layer of water-soluble polymer and a filter cake layer where suspended solids in the material solution are accumulated on the surface of the membrane. Suspended substances or water-soluble macromolecules are sterically hindered in the membrane pores, the surface adsorption of water-soluble macromolecules such as proteins in the membrane pores, and the salt precipitation of insoluble substances in the membrane pores may all be the cause of membrane blockage.

The pollution of the titanium rod filter element can be divided into two types: reversible pollution and irreversible pollution:

1) Reversible pollution. That is, after the filter element is contaminated, the flux can be restored after physical methods such as air washing, water washing, and ultrasonic cleaning. Air washing and water washing are usually performed online, while ultrasonic cleaning needs to be performed offline [7] [8] [9];

2) Irreversible pollution. That is, the filter element cannot be recovered by physical methods after being contaminated, and must be treated with chemical cleaning or even calcination. Chemical cleaning can usually be done online, while the calcination process must be done offline.

The pollution of seawater treatment by the titanium rod sintered filter element mainly comes from the following three aspects:

1) Suspended solids in seawater and tiny flocs and particles produced during seawater treatment;

2) Microorganisms and their dead bodies in seawater;

3) Inorganic salt in seawater crystallizes and scales on the filter element, causing filter hole blockage.

\subsection{Unblocking of Titanium Rod Filter Element}

The removal of the filter element mainly comes from physical and chemical methods.

Physical cleaning: mainly air blowing, water washing and ultrasonic cleaning.

Chemical cleaning: first strong acid washing, then strong alkali washing and then water washing, sometimes it is necessary to add strong oxidants. A lot of practice has proved that the cleaning sequence cannot be reversed, otherwise the 
cleaning effect will be worse [10] [11].

\section{Conclusions}

Microfiltration has two operation modes, namely dead-end filtration (terminal filtration) and cross-flow filtration (dynamic filtration). The former has a rapid and continuous decline in flux and a short operation period, while the latter has a flux that drops to a certain extent and stabilizes in a certain range, can implement continuous filtration operation for a long time to extend the backwash cycle of the filter element.

There are two typical mathematical models for filter element filtration, namely the filter cake filtration flux model and the flux attenuation model. Although the flux attenuation model is more effective in expressing the actual situation of microfiltration, its versatility is still poor and it has not been engineered.

The dead-end filtration test of the titanium rod sintered filter element shows that whether the filter element with different precision adopts constant pressure filtration or variable pressure filtration, the flux tends to be the same after a period of time. In most cases, the backwash time of the filter element should not exceed $2 \mathrm{~h}$, otherwise, the filtration flux is very small and seriously affects the production capacity. Constant flow filtration (or boosted filtration) is complicated to control, which is not good for backwashing filter elements and is not suitable for industrialization.

The pollution of the microporous filter element mainly comes from the surface adhesion of organic polymers, microorganisms, and inorganic salts, pore bridging, and pore blockage, which requires regular online backwashing and regular offline cleaning.

\section{Conflicts of Interest}

The authors declare no conflicts of interest regarding the publication of this paper.

\section{References}

[1] Chen, H. (2013) New Separation Technology. 2nd Edition, Chemical Industry Press, 59-62.

[2] Wang, Z. (2000) Fundamentals of Membrane Separation Technology. Chemical Industry Press, 269-271.

[3] Wang, X. (2013) Membrane Technology. Chemical Industry Press, 12-15.

[4] Zhang, X., Ge, H., et al. (2018) Analysis and Countermeasures of Filter Element Blockage of Security Filter. Shanghai Energy Conservation, Shanghai, 528-531.

[5] Liu, G. and Zuo, H. (2005) Analysis of the Damage of Stainless Steel Sintered Filter Element. Chemical Machinery, 32, 309-310.

[6] Zhang, J. (2019) RO Security Filter Filter Element Fouling Analysis and Solutions. New Technology Application and Practice, 169-170.

[7] Zhao, P. (2018) Ultrafiltration Membrane Cleaning and Pollution Prevention and Contro. Environmental Protection of Chemical Industry, 38, 333-337. 
[8] Li, X. and Lu H. (2018) Research Progress on Fouling and Cleaning of Ultrafiltration Membranes in the Yellow River Qing. Guangdong Chemical Industry, 45, 86-87.

[9] Yuan, T. (2015) Severe Pollution and Offline Cleaning of Ultrafiltration Membrane Elements. Energy and Environment, 4, 75-77.

[10] Li, Q. (2017) Research on the Chemical Cleaning of Ultrafiltration Membrane Pollution in Reclaimed Water Reuse. Clean World, 33, 30-34.

[11] Liu, K., Xu, D. and Ding, H. (2020) The Effect of Chemical Cleaning on the Performance and Membrane Fouling of Ultrafiltration Membranes of Different Materials. Water Technology, 14, 27-30. 